\title{
EVIDÊNCIAS DE VALIDADE INTERNA DA ESCALA DE REGULAÇÃO INTERNA NO ESPORTE
}

\author{
Evandro Morais Peixoto \\ Universidade de Pernambuco, Recife, Pernambuco, Brasil \\ Anna Catherine Graebin \\ Universidade La Salle, Canoas, Rio Grande do Sul, Brasil \\ Marcos Alencar Abaide Balbinotti \\ Université du Québec, Trois-Rivières, Quebec, Canadá \\ Leonardo Pestillo de Oliveira \\ Centro Universitário de Maringá, Maringá, Paraná, Brasil \\ Tatiana de Cássia Nakano \\ Centro de Ensino Superior de Maringá, Maringá, Paraná, Brasil \\ Ricardo Pedrozo Saldanha \\ Universidade La Salle, Canoas, Rio Grande do Sul, Brasil
}

\begin{abstract}
Resumo
O objetivo dessa pesquisa é estimar evidência de validade com base na estrutura interna e precisão da ERICEE-24, administrada em 261 estudantes do ensino fundamental de escolas públicas de Porto Alegre/RS (57,1\% do sexo feminino), com idades entre 12 e 15 anos (M= 13,36; DP =1,10). Análises fatoriais possibilitaram estimar a estrutura hierárquica do modelo de medida: um indicador global do construto Regulação Interna, refletindo quatro fatores específicos de primeira ordem (Orientação à Realização Pessoal-ORP; Orientação à Superação Pessoal-OSP; Orientação à Curiosidade Pessoal-OCP; Orientação à Estimulação PessoalOEP) que apresentaram índices de consistência interna que variam entre 0,82 e 0,84, e 0,91 para a escala total. Tais resultados podem ser entendidos como importante passo na direção da dissociabilidade empírica desse construto. Limitações da pesquisa foram discutidas.

Palavras-chave: Motivação. Psicologia do Esporte. Esporte para Jovens. Confiabilidade e Validade.

\section{EVIDENCE OF INTERNAL VALIDITY OF THE SCALE OF INTERNAL REGULA- TION IN SPORT}

\begin{abstract}
The objective of this research is to estimate evidence of validity based on the internal structure and accuracy of ERICEE-24, administered in 261 students from elementary schools of public schools in Porto Alegre / RS (57.1\% female), aged 12 and 15 years $(\mathrm{M}=13.36, \mathrm{SD}=$ 1.10). Factorial analyzes made it possible to estimate the hierarchical structure of the measurement model: a global indicator of the Internal Regulation construct, reflecting four specific
\end{abstract}


first order factors, showed internal consistency indexes ranging from 0.82 to 0.84 and 0.91 for the scale total. Such results can be understood as an important step towards the empirical dissociability of this construct. Limitations of the research were discussed.

Keywords: Motivation. Sport Psychology. Sport for Young People. Reliability and Validity.

\title{
EVIDENCIAS DE VALIDEZ INTERNA DE LA ESCALA DE REGULACIÓN IN- TERNA EN EL DEPORTE
}

\begin{abstract}
Resumen
El objetivo de esta investigación es examinar la evidencia de validez con base en la estructura interna y precisión de la ERICEE-24, administrada en 261 estudiantes de la enseñanza fundamental de escuelas públicas de Porto Alegre / RS (57,1\% sexo femenino), con edades entre 12 y 15 años $(\mathrm{M}=13,36, \mathrm{DP}=1,10)$. Los análisis factoriales permitieron estimar la estructura jerárquica del modelo de medida: un indicador global del constructo Regulación Interna, reflejando cuatro factores específicos de primer orden (Orientación a la Realización PersonalORP; Orientación a la Superación Personal-OSP; Orientación a Curiosidad Personal-OCP; la Estimulación Personal-OEP), presentaron índices de consistencia interna que variaban entre 0,82 y 0,84, y 0,91 para la escala Total. Esos resultados pueden ser entendidos como un importante paso hacia la disociabilidad empírica de este constructo. Se discutieron las limitaciones de la investigación.
\end{abstract}

Palabras clave: Motivación. Psicología del Deporte. Deporte para Jóvenes. Confiabilidad y Validez.

\section{Introdução}

Recentemente, alguns autores têm explicado o papel do comportamento autodeterminado no contexto da educação física e do esporte escolar (VAN DE BERGHE et al., 2014; VIANA; ANDRADE; MATIAS, 2010; FONTANA et al., 2013). Sabe-se que a Teoria da Autodeterminação (TAD) é considerada uma base teórica de impacto para estudos em educação física e esporte escolar (DE MEYER, et al., 2013; ROUSE; NTOUMANIS; DUDA, 2013; ROMERO et al., 2017), bem como para aqueles sobre a motivação no contexto em atividades físicas e esportivas (SCHNEIDER; KWAN, 2013). Os principais organizadores dessa teoria vêm relatando nesses últimos trinta anos (DECI; RYAN, 1985, 2013) que a motivação intrínseca é fundamentalmente regulada de forma interna, esses relatos se comprovam principalmente pelo desenvolvimento de instrumentos de avaliação da motivação intrínseca, ou de suas dimensões latentes, em diversos contextos. No entanto, nenhum instrumento foi desenvolvido para avaliar como os aspectos da regulação interna influenciam a motivação para a prática de atividade física no contexto escolar brasileiro.

A TAD entende o ser humano como organismo ativo e dirigido para o crescimento, pelos vieses do autodesenvolvimento e da integração com as estruturas sociais. Pode ainda ser entendida como uma teoria integradora geral (DECI; RYAN, 2013), organizada na forma de uma metateoria (ou macroteoria), composta por cinco outras teorias (ou mini teorias): (1) Teoria da Avaliação Cognitiva (TAC), a qual postula que aspectos ambientais suportam e incrementam a motivação intrínseca; (2) Teoria da Integração Orgânica (TIO), a qual postula que comportamentos extrinsecamente motivados podem ser internalizados e integrados ao repertório de comportamentos que satisfazem as necessidades básicas do indivíduo; (3) Teoria da Orientação Causal (TOC), a qual postula que, quando indivíduos regulam-se a si próprios, é porque apresentam uma orientação causal autônoma; (4) Teoria das Necessidades Básicas (TNB), a qual postula que as necessidades psicológicas básicas (de autonomia, de competên- 
cia e de pertença) são os nutrientes essenciais para o crescimento, integridade e bem estar dos indivíduos; e, finalmente, (5) Teoria da Orientação por Metas (TOM), a qual postula que a natureza das metas de uma pessoa, em uma atividade, explica sua motivação em uma atividade.

Figura 1 - Esquema dimensional da Teoria da Autodeterminação e suas componentes

AUTODETERMINAÇÃO

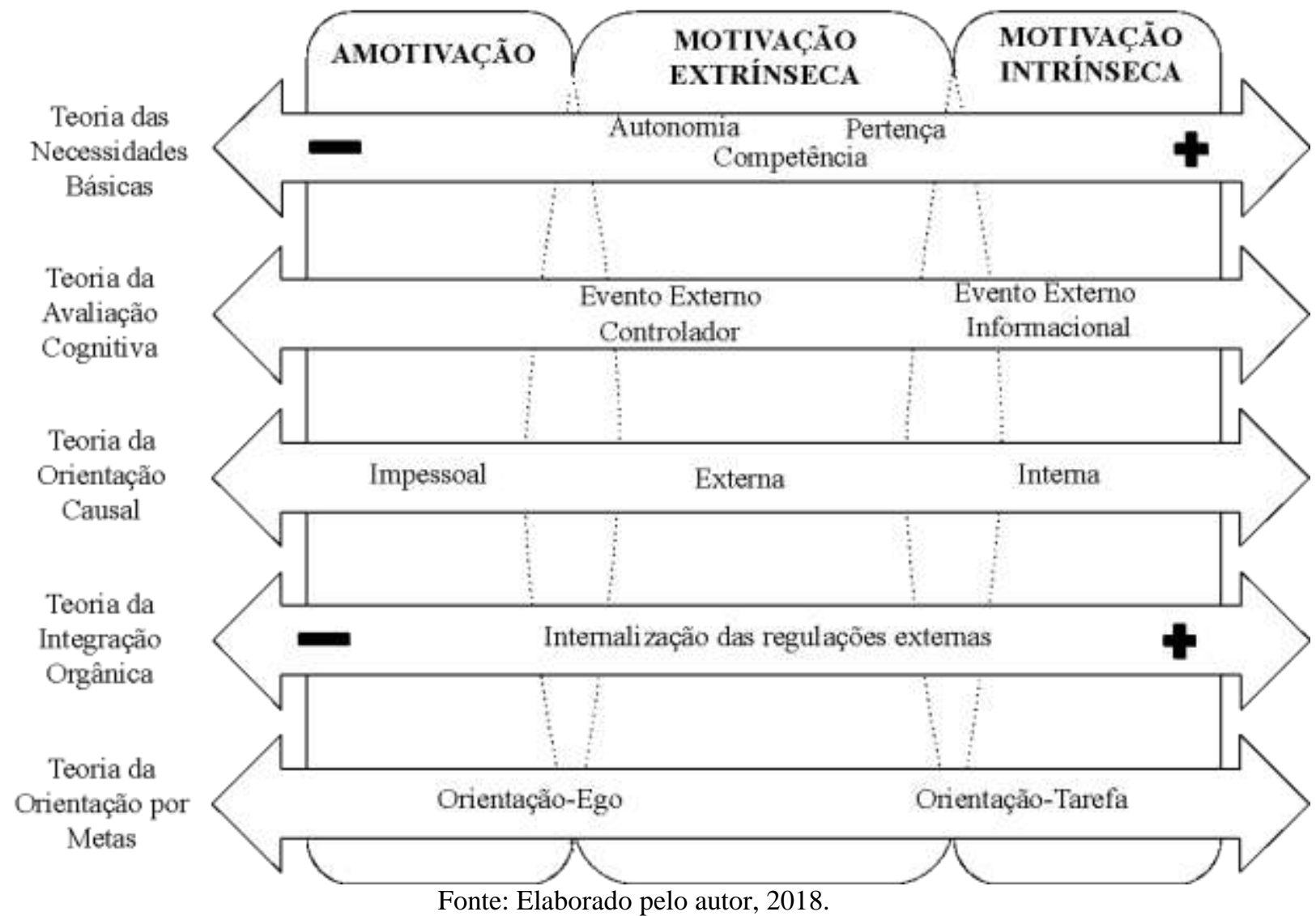

Analisando em detalhe esse esquema geral (Figura 1), pode-se entender que a busca de experiência com atividades interessantes estaria incluída no empenho evolutivo a fim de o indivíduo alcançar determinados objetivos, como desenvolver habilidades e exercitar capacidades, buscar e estabelecer vínculos sociais e obter um sentido unificado do self por meio da integração das experiências intrapsíquicas e interpessoais (RYAN; DECI, 2007; VAN DE BERGHE et al., 2014). A proposta da TAD é de desenvolver um olhar diferenciado sobre a motivação que se traduz em um continuum de diferentes processos regulatórios nos quais os resultados relacionados a uma atividade são exercidos, e em que os indivíduos têm um desejo inato para a estimulação e aprendizagem desde a nascença, que é reforçado ou desencorajado pelo meio social (DECI; RYAN, 2013; PELLETIER; SARRAZIN, 2007; RYAN; DECI, 2007). Seus estilos regulatórios, alocados em continuun de autonomia e de grau de internalização (DECI; RYAN, 2013), apresentam características que variam desde a falta de um propósito à ação (Amotivação) até a ação fundamentalmente autodeterminada (Motivação Intrínseca), passando pelas regulações "externa", "introjetada", "identificada" e "integrada" (Motivação Extrínseca).

Vallerand $(2004$; 2007) descreve a Amotivação como uma ausência total de finalidade ou intencionalidade em realizar uma ação. Já as características da Motivação Extrínseca le- 
vam o sujeito a não se dedicar a uma atividade por prazer, mas sim com o intuito de obter algum tipo de recompensa externa à sua própria vontade. A Regulação Externa é considerada o nível mais baixo de autodeterminação, em que o indivíduo se comporta simplesmente para obter alguma recompensa ou evitar uma punição (BRIÈRE; VALLERAND; BLAIS; PELLETIER, 1995). A Regulação Introjetada caracteriza-se por uma representação interna dos chamados contingentes externos, o que gera uma pressão do indivíduo sobre si mesmo (DECI; RYAN, 1985). A Regulação Identificada gera no indivíduo uma identificação com as consequências e o comportamento que é valorizado e considerado importante para o indivíduo, proveniente de sua própria escolha. Esse estilo pode ser observado quando uma ação ou um comportamento é motivado pela apreciação dos resultados e dos benefícios que a participação em determinada atividade pode gerar (BRIÈRE et al., 1995). A Regulação Integrada é considerada a forma mais autodeterminada de um comportamento e se caracteriza por um comportamento motivado extrinsecamente pois é realizado com vista à concretização de objetivos pessoais e não por um prazer em se envolver na atividade (DECI; RYAN, 1985).

Recentemente, alguns autores (CARBONNEAU; VALLERAND; LAFRENIÈRE, 2012) têm discutido a questão da indissociabilidade e da dissociabilidade da regulação interna, citando que, desde os anos 1950, os autores têm discutido a possibilidade de a Motivação Intrínseca (MI) se diferenciar em motivos específicos, sugerindo a existência de diferentes tipos. Essa ideia de multidimensionalidade da MI surge com White (1959) e Deci (1975), que discutem como a motivação se modifica no sujeito ao longo de seu desenvolvimento. Os autores argumentam que, enquanto bebês, a motivação intrínseca é geral e indiferenciada, se modificando gradualmente nas crianças, quando começam a se comportar de forma mais seletiva e desenvolver preferências por atividades específicas. Assim, ao acumular experiências, a MI se torna diferenciada em motivos específicos, no entanto, os autores (WHITE, 1959; DECI, 1975) não especificam a natureza dos tipos específicos de MI (CARBONNEAU; VALLERAND; LAFRENIÈRE, 2012).

Na tentativa de especificar os diferentes tipos de MI, alguns estudos propuseram a existência de categorias diferentes da MI, que apresentam a mesma origem, porém com diferentes antecedentes e consequências. Neste sentido, Carbonneau, Vallerand e Lafrenière (2012) propuseram em seu estudo testar um modelo com 3 tipos de Motivação Intrínseca, por meio de quatro argumentações: o primeiro argumento é o de que a MI pode ser considerada como um conceito multidimensional, isto porque no decorrer do desenvolvimento do sujeito, por meio do acúmulo de experiências, a base indiferenciada da MI se tornaria diferenciada, com motivos intrínsecos específicos; no entanto, essa natureza específica da MI ainda não foi especificada. O segundo argumento é o de que as mesmas condições que foram demonstradas na literatura para a origem da MI, devem ser consideradas para a origem de cada tipo da MI, ou seja, as variáveis que tipicamente influenciam a MI geral devem similarmente influenciar cada um dos tipos da MI. O terceiro argumento é que cada tipo de MI também apresenta seus antecedentes específicos, tais como, a personalidade, as atividades e as situações. Por fim, o quarto argumento é que um tipo específico de MI deve ser um melhor preditivo de consequências relevantes para esse tipo de MI do que os outros tipos.

Na realidade, Vallerand (2001) já havia proposto a existência de três estilos regulatórios latentes na motivação intrínseca: 1) conhecimento, 2) realização e 3) estimulação. O primeiro tipo de regulação (Conhecimento) é representado por muitos conceitos, tais como a exploração, a curiosidade, os objetivos intrínsecos de aprender, a motivação intrínseca intelectual, o desejo de conhecer e de saber, e a busca do significado. O segundo estilo regulatório (Realização) é composto por conceitos tais como: eficácia, motivação pela maestria da tarefa e uma orientação relacionada à tarefa, como forma de demonstrar a si mesmo que é competente, interagindo com o ambiente a fim de criar realizações únicas. Já o terceiro estilo regula- 
tório (Estimulação) está presente nas pessoas que fazem uma atividade pelo simples prazer por ela gerado, por apreciar a atividade (DECI; RYAN, 1985, 2013).

No sentido de discutir como a MI pode ser aplicada ao comportamento humano a partir da TAD, alguns instrumentos de medida são desenvolvidos com o intuito de demonstrar empiricamente as características da MI em diversos contextos, principalmente o esportivo. Alguns destes instrumentos são: General Causality Orientations Scale (GCOS - [DECI; RYAN, 1985]); b) Exercise Self-Regulation Questionnaire (SRQ-E - [DECI; RYAN, 2004]); c) Intrinsic Motivation Inventory (IMI - [MCAULEY; DUNCAN; TAMMEN, 1987]); d) The Self-Determination Scale (SDS - [SHELDON; DECI, 1996]); e) Motives for Physical Activity Measure (MPAM - [RYAN et al., 1997]); f) Sport Motivation Scale-II (SMS-II [PELLETIER et al., 2013]) ${ }^{1}$. No entanto, todos esses instrumentos avaliam a Motivação Intrínseca como um construto unidimensional, sem apresentar fatores específicos. A partir dessa lacuna presente na literatura, este estudo se faz necessário com o intuito de demonstrar como a MI pode ser considerada um construto multidimensional para se avaliar a motivação para a prática esportiva no contexto escolar brasileiro. Sendo assim, a Motivação Intrínseca seria avaliada a partir de quatro dimensões, a partir das características presentes na Teoria da Autodeterminação, as quais seriam: orientação à realização pessoal (ORP), orientação à superação pessoal (OSP), orientação à curiosidade pessoal (OCP), e orientação à estimulação pessoal (OEP). O objetivo desta pesquisa, então, é estimar evidência de validade com base na estrutura interna e precisão da Escala de Regulação Interna no Contexto do Esporte Escolar (ERICEE), um modelo hipotético de avaliação da regulação interna no contexto do esporte escolar, baseado na Teoria da Autodeterminação (TAD).

\section{Método}

\section{Participantes}

A amostra por conveniência foi composta por 261 adolescentes, estudantes do ensino fundamental de escolas públicas localizadas na região metropolitana de Porto Alegre, de ambos os sexos (57,1\% do sexo feminino) e como idades entre 12 e 15 anos $(\mathrm{M}=13,36$; $\mathrm{DP}=$ $1,10)$. Quanto ao ano escolar, eles se distribuem da seguinte maneira $6^{\circ}(28.4 \%) ; 7^{\circ}(29,1 \%)$; $8^{\circ}(39,1 \%)$ e $9^{\circ}(3,4 \%)$, dentre os quais $67,8 \%$ praticam alguma atividade física regular fora da escola.

\section{Instrumento}

Escala de Regulação Interna no Contexto do Esporte Escolar (ERICEE-24). Essa escala é composta por 24 itens que avaliam a regulação interna presente na prática esportiva, no contexto escolar (BALBINOTTI, 2016). Os itens estão divididos em 4 dimensões, Orientação à Realização Pessoal (ORP); Orientação à Superação Pessoal (OSP); Orientação à Curiosidade Pessoal (OCP); Orientação à Estimulação Pessoal (OEP), e são avaliados a partir de uma escala tipo Likert, de 5 pontos, indo de (1) Discordo Fortemente, (2) Discordo, (3) Não Concordo nem Discordo, (4) Concordo, à (5) Concordo Fortemente.

\section{Procedimentos e análises estatísticas}

Na busca de evidências de validade com base na estrutura interna da ERICEE-24 realizou-se, inicialmente, uma Análise Fatorial Exploratória (AFE), método de estimação dos

\footnotetext{
${ }^{1}$ Ver: <http://www.selfdeterminationtheory.org/questionnaires/>.
} 
mínimos quadrados não-ponderados (Unweighted Least Squares) e rotação Promax. Para a retenção do número de fatores, recorreu-se aos métodos Kaiser (soma dos quadrados das cargas), Cattell (Scree plot) e Análise Paralela (LORENZO-SEVA; TIMMERMAN; KIERS, 2011).

Tendo em vista a incompatibilidade dos resultados obtidos através dos métodos de retenção descritos anteriormente, recorreu-se a uma Análise Fatorial Confirmatória (AFC), sendo testados os índices de ajustes, bem como as cargas fatoriais do modelo unifatorial, conforme sugerido pelo Scree plot, bifatorial sugerido pela Análise Paralela, a estrutura com 4 fatores, conforme extraído pelo método Kaiser, assim Tendo em vista a incompatibilidade dos resultados obtidos através dos métodos de retenção descritos anteriormente, recorreu-se a uma Análise Fatorial Confirmatória (AFC). Foram testados: (a) os índices de ajustes; (b) as cargas fatoriais do modelo unifatorial, conforme sugerido pelo Scree plot; (c) bifatorial sugerido pela Análise Paralela; a estrutura com 4 fatores, conforme extraído pelo método Kaiser; (d) modelo hierárquico de segunda ordem, hipotetizado pelos autores deste estudo, que compreenderia uma variável latente geral (Regulação Interna) que tem como indicadores 4 fatores específicos (extraídos pelo método Kaiser). Para tanto, utilizou-se o método de estimação Weighted Least Square (WLSMV), a partir da matriz de correlações policóricas - haja vista que o WLSMV é um estimador robusto, mais adequado ao nível de mensuração ordinal, normalmente utilizada nas escalas psicométricas (BROWN, 2006). Dessa forma, os modelos foram testados a partir dos índices recomendados por L. Muthén e B. Muthén (2012), são estes: WLSMV $\chi^{2}$, df, RMSEA, CFI e TLI. Também se verificaram os índices de consistência interna do instrumento através dos coeficientes Alpha de Cronbach $(\alpha)$. Tais análises foram realizadas com o apoio do pacote estatístico MPlus versão 7.3 (MUTHÉN, L.; MUTHÉN, B. 2012; KANDAUDA et al., 2016).

Uma vez estabelecida a estrutura interna da ERICEE-24, avaliou-se a invariância configural, métrica e escalar do modelo de medida (BORSBOOM, 2006; MILFONT; FISHER, 2010) entre os grupos formados por adolescentes do sexo feminino e masculino. O teste da invariância dos parâmetros do modelo tem se mostrado cada vez mais essencial à psicologia, haja vista que os construtos psicológicos são avaliados em função de respostas a variáveis observadas. Dessa forma, ter evidências empíricas de que essas variáveis observadas estão relacionadas aos construtos latentes da mesma maneira para os diferentes grupos torna-se um pré-requisito para a comparação dos mesmos diante dos resultados brutos de um instrumento psicológico (BORSBOOM, 2006; MILFONT; FISHER, 2010). Isso se dá pois a violação desse pré-requisito toca diretamente às questões éticas ligadas à utilização dos testes (HUTZ, 2009).

Salienta-se que esta pesquisa ocorreu após a aprovação do projeto pelo Comitê de Ética da Universidade La Salle (CAAE 24916013.5.0000.5307). O instrumento foi administrado de forma coletiva, em sala de aulas, antecedido da assinatura do Termo de Consentimento Livre e Esclarecido por parte dos responsáveis, e do assentimentos dos adolescentes.

\section{Resultados}

A AFE foi antecedida pela verificação da medida de adequação da matriz de correlação através dos índices Kaiser-Meyer-Olkin $(\mathrm{KMO}=0,94)$, Teste de Esfericidade de Bartlett $\left(\chi^{2}{ }_{(276)}=3149,69 ; p<0,01\right)$ e o Determinante da Matriz de Correlação $\left(5,03^{-004}\right)$, indicando satisfatória adequação dos dados disponíveis aos modelos fatoriais testados. Com base nestes resultados, efetuou-se uma AFE, obtendo-se uma solução com quatro fatores, todos com valores próprios (eigenvalues) superiores a um, e total de variância explicada igual a 50,1\%. Resultados detalhados estão apresentados na Tabela 1. 
Tabela 1 - AFE dos resultados da ERICEE-24 com jovens (ambos os sexos) entre 12 e 15 anos ( $n=$ 261).

\begin{tabular}{|c|c|c|c|c|c|c|c|}
\hline \multirow[t]{2}{*}{ Dimensão } & \multirow[t]{2}{*}{ Item } & \multirow[t]{2}{*}{ Descrição resumida do conteúdo } & \multirow[t]{2}{*}{$h^{2}$} & \multicolumn{4}{|c|}{$\begin{array}{l}\text { Matriz fatorial } \\
\text { em quatro fatores }\end{array}$} \\
\hline & & & & OSP & ORP & OCP & OEP \\
\hline \multirow{6}{*}{$\begin{array}{l}\text { Orientação } \\
\text { à Superação } \\
\text { Pessoal } \\
(\mathrm{OSP})\end{array}$} & 07 & ...fazer certos movimentos difíceis & ,573 & ,726 & & & \\
\hline & 19 & ...executar com sucesso jogadas arriscadas & ,487 & ,723 & & & \\
\hline & 06 & ...explorar a determinação que tenho &, 512 & 663 & & & \\
\hline & 18 & ...se alegrar ao executar uma jogada difícil &, 588 & 647 & & & \\
\hline & 13 & ...efetuar uma técnica trabalhosa &, 552 & 643 & & & \\
\hline & 16 & ...executar golpes nunca antes realizados & 286 &, 551 & & & \\
\hline \multirow{6}{*}{$\begin{array}{c}\text { Orientação } \\
\text { à Realiza- } \\
\text { ção Pessoal } \\
\text { (ORP) }\end{array}$} & 17 & ...saber que o esporte é parte de minha vida & 557 & & ,766 & & \\
\hline & 28 & ...saber que sem o esporte eu não seria feliz & 384 & &, 528 & & \\
\hline & 05 & ...ficar feliz quando estou no treino &, 590 & &, 522 & & \\
\hline & 15 & ...praticar me deixa contente & ,569 & &, 520 & & \\
\hline & 14 & $\begin{array}{l}\text {...aprofundar meus conhecimentos no espor- } \\
\text { te }\end{array}$ & 484 & & ,499 & & \\
\hline & 08 & $\begin{array}{l}\text {...ter a sensação de bem-estar após um trei- } \\
\text { no }\end{array}$ & 460 & & ,477 & & \\
\hline \multirow{6}{*}{$\begin{array}{l}\text { Orientação } \\
\text { à Curiosi- } \\
\text { dade Pesso- } \\
\text { al (OCP) }\end{array}$} & 01 & ...descobrir novas formas de fazer jogadas & 648 & & & ,872 & \\
\hline & 04 & ...aprender melhores formas de jogar & ,495 & & & ,707 & \\
\hline & 10 & ...saber jogar melhor & ,392 & & &, 524 & \\
\hline & 02 & ...descobrir novas táticas &, 507 & & & 475 & \\
\hline & 03 & ...executar novas jogadas que dão certo &, 521 & & & ,473 & \\
\hline & 11 & $\begin{array}{l}\text {...descobrir novas formas de executar uma } \\
\text { jogada }\end{array}$ &, 562 & & & ,403 & \\
\hline \multirow{6}{*}{$\begin{array}{c}\text { Orientação } \\
\text { à Estimula- } \\
\text { ção Pessoal } \\
\text { (OEP) }\end{array}$} & 24 & ...ficar feliz o resto do dia & 479 & & & & 649 \\
\hline & 21 & ...sentir satisfação quando jogo bem &, 505 & & & & ,638 \\
\hline & 22 & ...sentir prazer quando ganho & 479 & & & & ,530 \\
\hline & 09 & ...ficar entusiasmado quando jogo bem & 609 & & & & , 487 \\
\hline & 20 & ...sentir a diversão que o jogo oferece &, 539 & & & & 420 \\
\hline & 23 & $\begin{array}{l}\text {...ter satisfação quando estou jogando me- } \\
\text { lhor }\end{array}$ & ,374 & & & & ,401 \\
\hline \multicolumn{4}{|c|}{ Porcentagem de variância por fator - valores extraídos } & 39,94 & 4,83 & 3,23 & 2,76 \\
\hline \multicolumn{4}{|c|}{ Alpha de Cronbach por fator } & 0,88 & 0,84 & 0,83 & 0,81 \\
\hline
\end{tabular}

Fonte: Elaborado pelo autor, 2018.

Obs.: Método de extração ULS ("Unweighted Least Squares"), com rotação Promax; Comunalidades $=h^{2}$; rotação convergida em 9 interações; saturações fatoriais menores de 0,40 não estão apresentadas na Tabela 1.

Em relação aos agrupamentos de itens nos respectivos fatores, estes apresentam conteúdos que avaliam, no primeiro fator, a Orientação à Realização Pessoal (ORP) (por exemplo, item 05: "pelo prazer que sinto quando estou praticando"); no segundo fator, a Orientação à Superação Pessoal (OSP) (por exemplo, item 19: "pela empolgação que sinto ao executar com sucesso certos movimentos difíceis"); no terceiro fator, a Orientação à Curiosidade Pessoal (OCP) (por exemplo, item 01: "por me sentir entusiasmado quando aprendo novas formas de fazer uma jogada"); e no quarto fator, a Orientação à Estimulação Pessoal (OEP) (por exemplo, item 22: "pela sensação de prazer que eu sinto quando ganho"). Em relação às cargas fatoriais observou-se que todos os itens apresentaram índices superiores a 0,40, com exceção 
do item 23 (0,292). Além disso, verificou-se que apenas o item 14 apresentou dupla saturação, nos fatores $\mathrm{ORP}=0,579$ e $\mathrm{OEP}=0,361$. No entanto se optou pela manutenção dos dois itens haja vista a importância do conteúdo avaliado pelo item 23 ("pela diversão que a competição oferece"), bem como a adequação teórica do item 14 ("pelo interesse que sinto em aprofundar meus conhecimentos sobre meu esporte") ao fator ORP, que obteve carga significativamente mais alta.

Contudo, através da análise gráfica Scree plot pode-se observar a indicação da existência de apenas um fator. Recorreu-se então à Análise Paralela, que demonstrou a pertinência da extração de dois fatores. Os resultados desses métodos são comparados na Figura 2, em que se observa que apenas os dois primeiros autovalores dos dados reais são superiores aos obtidos aleatoriamente, são estes: 10,019; 1,575; 1,244 (para os dados reais); 1,$593 ; 1,495 ; 1,422$ (para a média dos dados aleatórios); e 1,689; 1,570; 1,478 (para os alocados nos postos percentis superiores a 95).

Figura 2 - Análise Paralela

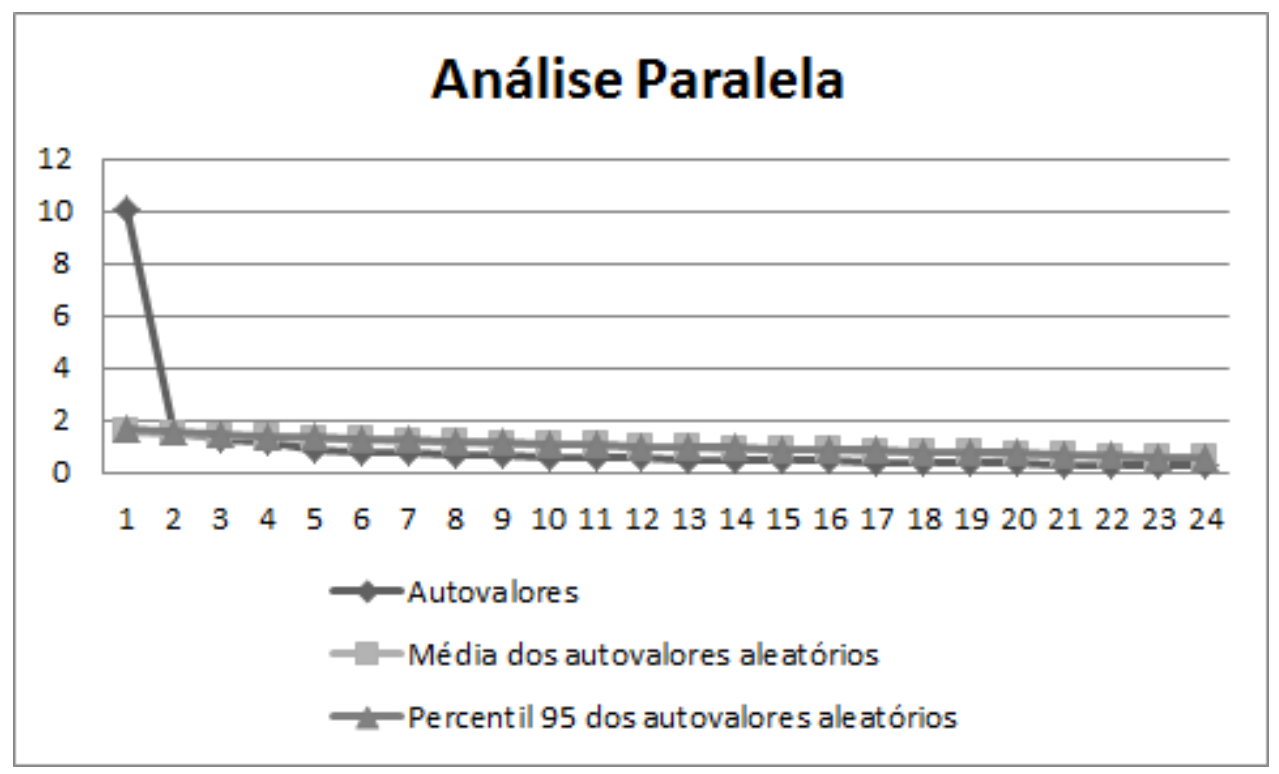

Fonte: Elaborado pelo autor, 2018.

Nota: A análise paralela consiste em um procedimento estatístico de simulação de dados que objetiva à construção aleatória de matrizes de correlação, para tanto se baseia nas mesmas características do conjunto de dados reais (número de participantes e número de variáveis). Assim, os autovalores obtidos através das matrizes simuladas (1000 matrizes) são comparados com os autovalores extraídos dos dados reais. Portanto, o número de dimensões a serem retidos nos dados reais corresponde ao número de autovalores que apresentam índices superiores àqueles obtidos por meio dos dados aleatórios (quando pareados). Existem dois métodos normalmente utilizados para a comparação desses valores, o valor médio dos autovalores produzidos aleatoriamente e os valores que se alocam nos postos percentis superiores a 95 (CRAWFORD, 2010).

Considerando a adequação teórica da solução com quatro fatores, bem como os resultados apontados pelos diferentes métodos de extração, optou-se por submeter os dados à AFC e ao teste de verificação dos índices de ajustes às diferentes soluções fatoriais, são elas: unidimensional, bidimensional, multidimensional (com quatro fatores), e hierárquica de segunda ordem, que compreende a Regulação Interna como um fator latente geral, de segunda ordem, seguida de quatro fatores de primeira ordem (aqueles indicados pelo método Kaiser), os quais, por sua vez, refletem suas respectivas variáveis observadas. Os índices de ajuste comparando esses modelos estão apresentados na Tabela 2. 
Tabela 2 - Índices de ajustes da análise fatorial confirmatória dos modelos estruturais da ERICEE-24

\begin{tabular}{lccccccc}
\hline Solução avaliada & $\chi^{2}$ & df & $\chi^{2} / \mathrm{df}$ & CFI & TLI & RMSEA & $\begin{array}{c}\text { RMSEA I. C } \\
90 \%\end{array}$ \\
\hline Unidimensional & 650,017 & 252 & 2,579 & 0,922 & 0,915 & 0,078 & $0,070-0,085$ \\
Bidimensional & 587,149 & 252 & 2,329 & 0,934 & 0,927 & 0,072 & $0,064-0,079$ \\
Multidimensional & 446,770 & 246 & 1,816 & 0,951 & 0,946 & 0,056 & $0,048-0,064$ \\
Hierárquica & 434,044 & 248 & 1,750 & 0,965 & 0,959 & 0,054 & $0,045-0,062$ \\
\hline
\end{tabular}

Fonte: Elaborado pelo autor, 2018.

A solução fatorial hierárquica, que apresentou melhor ajuste aos dados reais, é apresentada na Figura 3.

Figura 3 - Solução Fatorial Hierárquica para ERICEE-24.

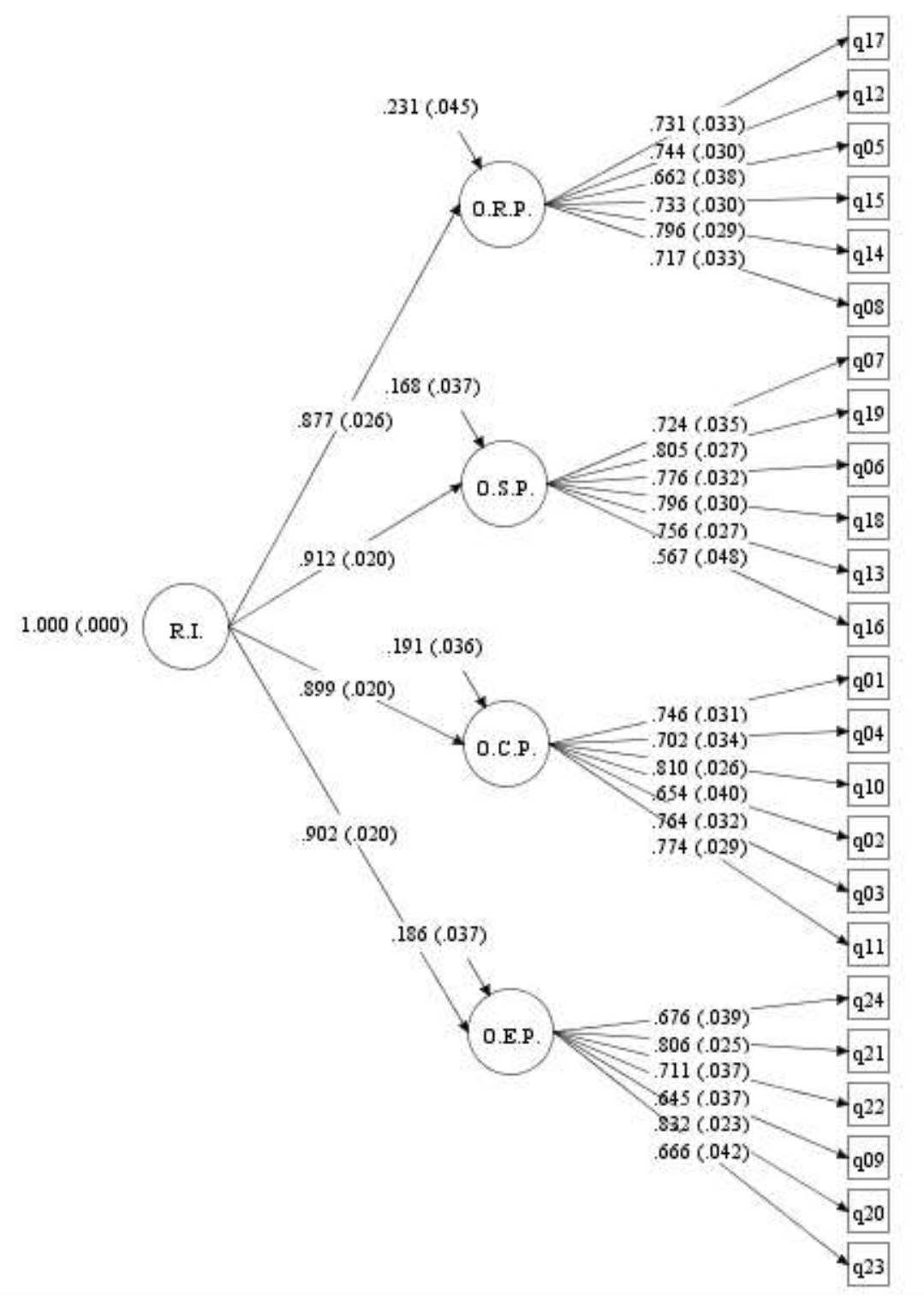

Notas: RI = Regulação Interna; ORP = Orientação à Realização Pessoal; OSP = Orientação à Superação Pessoal; $\mathrm{OCP}=$ Orientação à Curiosidade Pessoal; OEP = Orientação à Estimulação Pessoal. Fonte: Elaborado pelo autor, 2018 . 
De acordo com a Figura 3, observa-se que os itens apresentam elevadas cargas fatoriais em suas respectivas dimensões, superiores a 0,566, contudo a análise estima também as correlações entre a variável latente geral RI (Regulação Interna) e seus indicadores, fatores de primeira ordem: ORP, OSP, OCP e OEP, também consideradas elevadas, superiores a 0,876. Uma vez estabelecida a estrutura interna do instrumento testou-se a invariância do modelo entre os grupos de adolescentes do sexo feminino e masculino. Cabe ressaltar que apenas os fatores de segunda ordem podem ser testados diretamente no que diz respeito à invariância dos parâmetros (CHEN; WEST; SOUSA, 2006).

Os resultados desta análise demonstraram os seguintes índices de ajustes para o modelo configural $\left(\chi^{2}=690,609 ; \mathrm{gl}=448 ; \chi^{2} / \mathrm{gl}=1,541 ; \mathrm{CFI}=0.949 ; \mathrm{TLI}=0.943 ; \mathrm{RMESA}=0.064\right.$ I.C. $90 \%$ 0.055-0.074); modelo métrico $\left(\chi^{2}=696,784 ; \mathrm{gl}=467 ; \chi^{2} / \mathrm{gl}=1,492 ; \mathrm{CFI}=0.952\right.$; TLI $=0.948$; RMESA $=0.061$ I.C. $90 \%=0.052-0.071)$ e modelo escalar, $\left(\chi^{2}=763,268 ; \mathrm{gl}=\right.$ 532; $\chi^{2} / \mathrm{gl}=1,43 ; \mathrm{CFI}=0.952 ; \mathrm{TLI}=0.954 ; \mathrm{RMESA}=0.058$ I.C. $\left.90 \%=0.048-0.067\right)$. Assim, através do teste de invariância pode-se verificar que o modelo configural, estrutura proposta, é invariante e portanto apresenta bom ajustamento para ambos os grupos $(p=0.5647)$, assim como o modelo métrico, ou seja, pesos fatoriais $(p=0.0891)$. Contudo, verificou-se que as cargas fatoriais dos itens em seus respectivos fatores foram diferentes entre os grupos ( $p=$ 0.0167).

Por fim, estimaram-se os índices de precisão de cada um dos fatores que compreendem a segunda ordem hierárquica, assim como para a escala total. Os resultados obtidos foram: $\alpha=0,84$ para o fator ORP; $\alpha=0,84$ para 0.84 para OSP, $\alpha=82$ para OCP e $\alpha=81$ para OEP, e $\alpha=0,93$ para escala total.

\section{Discussão}

Entende-se que o modelo de medida proposto através da ERICEE traz uma importante contribuição à área de estudo, ao compreender a MI de forma hierárquica, visto que os resultados apresentam índices conforme o recomendado pela literatura e encontrado em estudos similares (REPPOLD; GURGEL; HUTZ, 2016).

Embora sustente a ideia de um indicador global do construto (fator de $2^{\mathrm{a}}$ ordem), esse fator emerge da associação dos fatores específicos (quatro fatores de primeira ordem: ORP, OSP, OCP e OEP), o que pode ser entendido como importante passo na direção da dissociabilidade empírica deste construto. Haja vista a proposta teórica de Vallerand (2001) em compreender os estilos regulatórios latentes na motivação intrínseca, vale ressaltar que tais perspectivas teóricas ainda não foram captadas pelos instrumentos de avaliação normalmente utilizados pelos pesquisadores da área, uma vez que estes avaliam a MI unidimensionalmente.

O fato de compreender o construto MI conforme avaliado pela ERICEE, hierarquicamente, traz benefícios também aos profissionais inseridos no contexto do esporte escolar (psicólogos do esporte, educadores físicos, pedagogos do esporte, entre outros) ao proporcionar conhecimentos específicos referentes à MI de seus alunos. Não se questiona aqui a importância de saber o quanto tais sujeitos são motivados (índice global), no entanto, salienta-se a relevância da informação a respeito de quais são os fatores que contribuem para o aumento ou rebaixamento desse índice global. Nessa direção, saber que um adolescente apresenta níveis elevados em determinadas características (por exemplo: ORP e OSP) e níveis mais baixos em outras (OCP e OEP) pode se caracterizar como uma informação importante ao desenvolvimento de ações interventivas adequadas. Com isso, podem ser elaboradas estratégias de treinamento que estimulem os adolescentes através de novos desafios, desde que sejam coerentes com o nível esportivo do adolescente, para que eles possam vivenciar o prazer em aprender novas formas de realizar os fundamentos do esporte. Ademais, pode-se estimular o desenvol- 
vimento de situações em que o adolescente possa experimentar a vitória, e, portanto, novos sentimentos relacionados à essa experiência. Essas ações mais específicas podem contribuir para o desenvolvimento do índice geral de MI dos adolescentes, proporcionando melhor relação com a prática esportiva.

Destaca-se ainda a capacidade da escala em avaliar adolescentes do sexo feminino e masculino de maneira semelhante, o que possibilita aos pesquisadores da área realizar comparações entre os grupos sem infringir questões éticas, ou seja, sem o conhecimento das potencialidades do instrumento para este fim (BORSBOOM, 2006; MILFONT; FISHER, 2010). Contudo, considerando que o processo de obtenção de evidências de validade de um instrumento pode exigir diversos estudos, sugere-se que novas pesquisas sejam realizadas com tais objetivos, haja vista que a AFC não foi utilizada com o objetivo de testar a estabilidade (validação cruzada) do instrumento em uma amostra diferente, mas sim como um procedimento que possibilitou a estimação do modelo fatorial mais adequado. Além disso, faz-se necessário o desenvolvimento de normas interpretativas da ERICEE que possibilitem interpretação dos escores brutos apresentados pelos sujeitos avaliados pela escala. Dessa maneira, disponibilizar-se-á aos profissionais e acadêmicos da área um instrumento desenvolvido especificamente para a realidade brasileira.

Por fim, cabe salientar que outras medidas de avaliação (autoeficácia, autoestima etc.), bem como outras metodologias que não se restrinjam aos testes psicológicos são particularmente necessárias quando utilizadas dentro do contexto da avaliação psicológica, garantindo ao sujeito uma avaliação mais integradora, composta por diferentes vertentes, de modo que se constitua a mais completa e abrangente possível (ANASTASI; URBINA, 2000). Isso, principalmente, quando o objetivo dos profissionais interessados (psicólogos do esporte, treinadores, professores de educação física, entre outros) é coletar informação para se compreender melhor o desenvolvimento do atleta.

\section{Conclusões}

Considerando que o objetivo desta pesquisa foi estimar as primeiras evidências de validade e precisão da ERICEE, pode-se assumir que tais objetivos foram satisfatoriamente alcançados, uma vez que os métodos utilizados AFE e AFC possibilitaram estimar a estrutura interna mais adequada ao modelo de medida. Além disso, os índices de confiabilidade alfa de Cronbach demonstraram a precisão do instrumento, bem como de suas dimensões.

A partir desta conclusão, pode-se supor que esse instrumento pode ser aplicado em contextos diminutos, abrindo caminho para novas investigações e resultados importantes para a prática. Esse instrumento pode ser útil aos professores de educação física, visto que o modelo hierárquico da Motivação Intrínseca (MI) possibilitará uma compreensão mais detalhada sobre o comportamento autodeterminado de seus alunos, consequentemente favorecendo um melhor planejamento de suas aulas e intervenções. Além disso, vale ressaltar algumas das limitações do presente estudo, como o fato de contar com uma amostra por conveniência oriunda de uma região específica do país, região Sul. Em pesquisas futuras esforços devem ser realizados no sentido de envolver amostras mais representativas geograficamente e, portanto, maiores, possibilitando o estudo de invariância do modelo fatorial em função da idade dos participantes ou séries de estudo, haja vista que pesquisadores normalmente buscam estimar diferentes aspectos psicológicos entre alunos em função dessas características.

\section{Referências}

ANASTASI, A.; URBINA, S. Testagem Psicológica. Porto Alegre: Artes Médicas Sul, 2000. 
BALBINOTTI, M. A. A. Escala de Regulação Interna no Contexto do Esporte Escolar (ERICEE-24). Laboratório de Psicologia do Esporte - Universidade Federal do Rio Grande do Sul: Porto Alegre, 2016.

BORSBOOM, D. The attack of the psychometricians. Psychometrika, v. 71, n. 3, p. 425440, set. 2006.

BRIÈRE, N. M.; VALLERAND, R. J.; BLAIS, M. R. e PELLETIER, L. G. Développement et validation d'une mesure de motivation intrinsèque, extrinsèque et d'amotivation en context sportif: l'échelle de motivation dans les sports (ÉMS). International Journal of Sport Psychology, v. 26, n. 4, p. 465-489, 1995.

BROWN, T.A. Confirmatory factor analysis for applied research. New York: The Guilford Press, 2006.

CARBONNEAU, N.; VALLERAND, R.; LAFRENIERE, M. Toward a tripartite model of intrinsic motivation. Journal of Personality, v. 80, n. 5, p. 1147-1178, out. 2012.

CHEN F.F.; WEST, S. G.; SOUSA, K.H.A. Comparison of Bifactor and Second-Order Models of Quality of Life. Multivariate Behavioral Research, v. 41 n. 2, p.189-225, jun. 2006.

DE MEYER, J.; SOENENS, B.; AELTERMAN, N.; BOURDEAUDHUIJ, I.; HAERENS, L. The different faces of controlling teaching: implications of a distinction between externally and internally controlling teaching for students' motivation in physical education. Physical Education and Sport Pedagogy, v. 21, n.6, 632-652, 2016. doi:10.1080/17408989.2015.1112777

DECI, E. L. Intrinsic motivation. New York: Plenum, 1975.

DECI, E. L.; RYAN, R. M. Exercise self-regulation questionnaires: self-determination theory: an approach to human motivation and personality; the self-regulation questionnaires. 2004. Disponível em: <www.psych.rochester.edu/SDT/measures/selfreg_exer.html>.

Intrinsic motivation and self-determination in human behavior. New York: Plenum Press, 1985.

FONTANA, P. S. et al. Estudo das motivações à prática da ginástica rítmica: contribuições da pesquisa para o rendimento desportivo. Pensar a Prática, Goiânia, v. 16, n. 2, p. 320618, abr./jun. 2013.

HUTZ, C. S. Ética na avaliação psicológica. In: HUTZ, C. S. (Org.). Avanços e polêmicas em avaliação psicológica. São Paulo: Casa do Psicólogo, 2009. p. 297-310.

KANDAUDA K.A.S. et al. Higher-Order Growth Curves and Mixture Modeling with Mplus: A Practical Guide. New York: Routledge, 2016.

LORENZO-SEVA, U.; TIMMERMAN, M. E.; KIERS, H. A.L. The Hull Method for Selecting the Number of Common Factors. Multivariate Behavioral Research, v. 46, n. 2, p. 340364, abr. 2011. 
MILFONT, T.L.; FISHER, R. Testing measurement invariance across groups: Applications in cross-cultural research. International Journal of Psychological Research, v. 3, n. 1, p. 111$121,2010$.

MCAULEY, E.; DUNCAN, T.; TAMMEN, V. V. Psychometric properties of the Intrinsic Motivation Inventory in a competitive sport setting: a confirmatory factor analysis. Research Quartely for Exercise and Sport, v.60, n. 1, p. 48-58, mar. 1989.

MUTHÉN, L. K.; MUTHÉN, B.O. Mplus User's Guide. Seventh Edition. Los Angeles, CA, 2012.

PELLETIER, L.G.; SARRAZIN, P. Measurement issues in self-determination theory and sport. In: HAGGER, M. S.; CHATZISARANTIS, N. L. D. (Ed.). Intrinsic motivation and self-determination in exercise and sport. Champaign: Human Kinetics, IL, 2007. p. 143152.

PELLETIER, L. G.; ROCCHI, M. A.; VALLERAND, R. J.; DECI, E. L.; RYAN, R. M. Validation of the revised sport motivation scale (SMS-II). Psychology of Sport and Exercise, v. 14, n. 3, p. 329341, mai. 2013.

REPPOLD, C. T.; GURGEL, L. G.; HUTZ, C. S. Escala de Avaliação de Humor para Adolescentes: evidências de validade. Arquivos Brasileiros de Psicologia, Rio de Janeiro, v. 68, n. 2, p. 21-30, 2016.

ROMERO, B. F. et al. Motivação e atividade física: os projetos de extensão na universidade (motivação e atividade física). Pensar a Prática, Goiânia, v. 20, n. 1, p. 76-87, jan./mar. 2017.

ROUSE, P. C.; NTOUMANIS, N.; DUDA, J. L. Effects of motivation and depletion on the ability to resist the temptation to avoid physical activity. International Journal of Sport and Exercise Psychology, v. 11, p. 39-56, set. 2013.

RYAN, R.M.; DECI, E. L. Active human nature: Self-determination theory and the promotion and maintenance of sport, exercise, and health. In: HAGGER, M. S.; CHATZISARANTIS, N. L. D. (Ed.). Intrinsic motivation and self-determination in exercise and sport. Champaign, IL: Human Kinetics, 2007. p. 1-19.

SCHNEIDER, M.; KWAN, B. Psychological need satisfaction, intrinsic motivation and affective response to exercise in adolescents. Psychol. sport exerc., v. 4 n. 5, p. 776-785, 2013.

SHELDON, K. M.; RYAN, R. M.; REIS, H. What makes for a good day? Competence and autonomy in the day and in the person. Personality and Social Psychology Bulletin, v. 22, p. 1270-1279, dez. 1996.

VALLERAND, R. J. A hierarchical model of intrinsic and extrinsic motivation in sport and exercise. In: Roberts, G. C. Advances in Motivation in Sport and Exercise. Champaign: Human Kinetics, 2001. p. 263-319. 
A hierarchical model of intrinsic and extrinsic motivation for sport and physical activity. In: HAGGER, M. S.; CHATZISARANTIS, N. L. D. Intrinsic motivation and selfdetermination in exercise and sport. Champaign: Human Kinetics, IL, 2007. p. 255-279.

. Intrinsic and extrinsic motivation in sport. In: Encyclopedia of Applied Psychology, 2004, 2, p. 427-435. San Diego: Academic Press.

VAN DE BERGHE, L. et al. Research on self-determination in physical education: Key findings and proposals for future research. Physical Education and Sport Pedagogy, v. 1, p. 97121, nov. 2014.

VIANA, M.S, ANDRADE, A., MATIAS, T.S. Teoria da autodeterminação: aplicações no contexto da prática de exercícios físicos de adolescentes. Pensar a Prática, Goiânia, v. 13, n. 2, p. 118, maio/ago. 2010.

WHITE, R. W. Motivation reconsidered: The concept of competence. Psychological Review, v. 66, p. 297-333, 1959.

Recebido em: 13/10/2017

Revisado em: 28/05/2018

Aprovado em: 03/08/2018

Endereço para correspondência:

ricardo@ ricardosaldanha.com.br

Ricardo Pedrozo Saldanha

Universidade La Salle - Canoas.

Avenida Victor Barreto, 2288

Centro

92010000 - Canoas, RS - Brasil 